\title{
Defocused imaging of quantum-dot angular distribution of radiation
}

\author{
Digambara Patra, ${ }^{\text {a) }}$ Ingo Gregor, and Jörg Enderlein ${ }^{\text {b) }}$ \\ Institute for Biological Information Processing 1 \& 2, Forschungszentrum Jülich, \\ D-52425 Jülich, Germany

\begin{abstract}
Markus Sauer
Department of Physics, Applied Laserphysics and Laserspectroscopy, University of Bielefeld, Universitätsstr. 25, D-33615 Bielefeld, Germany
\end{abstract}

(Received 15 April 2005; accepted 12 July 2005; published online 29 August 2005)

\begin{abstract}
We have applied defocused imaging of single fluorescent quantum dots to study the angular distribution of their emission. It is found that quantum dots exhibit an angular distribution best described by a superposition of emission of three perpendicular dipoles. A theory of the defocused images of such emitters is presented and compared with the measurements. Furthermore, it is shown that standard fluorescence anisotropy measurements are not able to uncover such complex emission behavior. (C) 2005 American Institute of Physics. [DOI: 10.1063/1.2037194]
\end{abstract}

In recent years, fluorescent colloidal semiconductor nanocrystals (quantum dots) have attracted considerable interest. ${ }^{1}$ Although the fluorescence emission of the nanocrystals is stemming from a dipole transition, their angular distribution of emission differs from that of single dipole emitters with fixed dipole axis. ${ }^{2}$ In contrast to organic fluorophores with fixed emission dipole orientation, spherical nanocrystals, in particular CdSe nanocrystals, can have a degenerate transition dipole oriented isotropically in two dimensions, which gives rise to a perpendicular "dark axis" that does not couple to the light field. ${ }^{3}$ However, an elongation of the crystal shape along the extraordinary axis of the wurtzite-type crystal structure can result in an electronic structure that transforms the "dark axis" into the emitting dipole transition. ${ }^{2,4}$ In this letter, we study the angular distribution of such nanocrystals using defocused imaging. ${ }^{5}$ This method has proven to be very efficient in measuring the three-dimensional orientation of emission dipoles of single molecules, ${ }^{6}$ nanocrystals, ${ }^{7}$ dipole reorientation dynamics of molecules in polymer hosts, ${ }^{8}$ or in multichromophoric systems. ${ }^{9}$ It was shown that there is perfect correspondence between complete $a b$ initio electrodynamic modeling of defocused imaging and measurement. ${ }^{10}$ For calculating the defocused image of a perfect dipole emitter on the CCD detector, one has to calculate the position dependent energy flux through the detector plane, which is proportional to the perpendicular component of the electrodynamic Poynting vector through that plane. Assuming, for the moment, polarized fluorescence detection, i.e., the insertion of a polarizing filter in front of the detector, the perpendicular component of the Poynting vector is proportional to the product of the electric field component, $E_{x}$, times the complex conjugate of the magnetic field component, $B_{y}$, where the $x$ - and $y$-axes form an orthogonal coordinate system in the detector plane (perpendicular to the optical $z$-axis), and the $x$-axis is oriented along the polarizing direction of the polarization filter. As was shown in Refs. 5,10, the electric and magnetic field components are given by

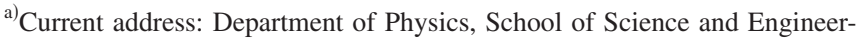
ing, Okubo 3-4-1, Shinjukuku, Tokyo, 169-8555, Japan.

${ }^{b}$ Author to whom correspondence should be addressed; electronic mail: j.enderlein@fz-juelich.de; Internet: http://www.joerg-enderlein.de
}

$$
\begin{aligned}
E_{x} \sim & {\left[F_{0}(\rho, \delta f) \cos \beta-F_{2}(\rho, \delta f) \cos (2 \phi-\beta)\right] \sin \alpha } \\
& +F_{1}(\rho, \delta f) \cos \phi \cos \alpha, \\
B_{y} \sim & {\left[G_{0}(\rho, \delta f) \cos \beta-G_{2}(\rho, \delta f) \cos (2 \phi-\beta)\right] \sin \alpha } \\
& +G_{1}(\rho, \delta f) \cos \phi \cos \alpha,
\end{aligned}
$$

where $\alpha$ is the angle between the dipole and the optical axis; $\beta$ is the angle between the $x y$-projection of the dipole and the $x$-axis; and $\rho=\left(x^{2}+y^{2}\right)^{1 / 2}, \phi=\arctan (y / x)$ and $z$ define the position (in cylindrical coordinates) where the fields are calculated. The $F_{j}$ and $G_{j}$ are integrals involving Bessel functions containing the complete information about the imaging properties of the optics (inclusively the image defocusing) as well as the modification of the angular distribution of emission of the imaged dipole due to its electrodynamic interaction with the substrate. ${ }^{11}$

In the present letter, defocused imaging is used for studying the angular distribution of emission of fluorescent CdSe quantum dots. Instead of assuming them to be perfect dipole emitters with one fixed orientation or with perfectly circular two-dimensional degeneracy, we will assume their emission to be a superposition of three perpendicular linear dipole emitters with differing emission intensity. The orientation of this dipole system with respect to the lab system can be defined by three Euler angles $\Psi, \Omega$, and $\omega$, as shown in Fig. 1. Knowing these three angles, one can calculate in a straightforward way the angles $\alpha$ and $\beta$ for each of the three

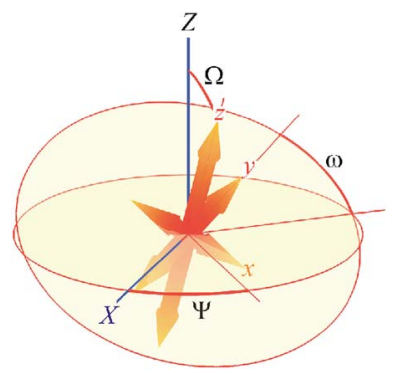

FIG. 1. (Color online) Geometric parameters determining the emission of a quantum dot. The three-dimensional orientation of the dot's reference system $\{x, y, z\}$ with respect to the lab system (indicated by $\{X, Z\}$ ) is determined by the three Eulerian angles $\Psi, \Omega$, and $\omega$. Optical axis is along the $Z$-axis, which points away from the imaging objective. 
dipoles, and using these angles the resulting Poynting vector following Eqs. (1)-(4). For calculating the correct superposition of the three defocused images, one has to know additionally the relative ratio of the emission intensities of the three dipoles. If we denote by $I_{x}, I_{y}$, and $I_{z}$ the intensity distributions of the defocused images of the three perpendicular dipoles (see Fig. 1) with unit emission strength, the final intensity distribution measured by a camera will be proportional to

$$
P=\kappa I_{z}+(1-\kappa)\left[\frac{1+\eta}{2} I_{y}+\frac{1-\eta}{2} I_{x}\right],
$$

where the two parameters $\kappa$ and $\eta$ define the intensity ratios of the three dipoles. The resulting expression can be developed into a finite series over the angular coordinate $\phi$,

$$
\begin{aligned}
& P(\rho, \phi, \Psi, \Omega, \omega, \kappa, \eta, \delta f) \\
& \quad=C_{0}+\sum_{m=1}^{4}\left(C_{m} \cos m \phi+S_{m} \sin m \phi\right),
\end{aligned}
$$

where the coefficients $C_{j}, S_{j}$ read

$$
\begin{aligned}
C_{0}= & (3-\kappa) F_{0} G_{0}^{*}+(1+\kappa) F_{1} G_{1}^{*}+(3-\kappa) F_{2} G_{2}^{*} \\
& -F_{0} G_{0}^{*} \cos 2 \Psi\left[2(1-3 \kappa) \sin ^{2} \Omega+\eta(1-\kappa)\right. \\
& \times(3+\cos 2 \Omega) \cos 2 \omega]+\left(F_{0} G_{0}^{*}-F_{1} G_{1}^{*}+F_{2} G_{2}^{*}\right) \\
& \times\left[(1-3 \kappa) \cos 2 \Omega+2 \eta(1-\kappa) \cos 2 \omega \sin ^{2} \Omega\right] \\
& +4 \eta(1-\kappa) F_{0} G_{0}^{*} \cos \Omega \sin 2 \Psi \sin 2 \omega,
\end{aligned}
$$$$
C_{1}=-2\left[\left(2 F_{0}-F_{2}\right) G_{1}^{*}+F_{1}\left(2 G_{0}^{*}-G_{2}^{*}\right)\right]
$$$$
\cdot \sin \Omega\{\cos \Psi \cos \Omega[1-3 \kappa-\eta(1-\kappa) \cos 2 \omega]
$$$$
+\kappa \sin \Psi \sin 2 \omega\} \text {, }
$$$$
C_{2}=-(3-\kappa) F_{2} G_{0}^{*}+(1+\kappa) F_{1} G_{1}^{*}-(3-\kappa) F_{0} G_{2}^{*}
$$$$
+\left(F_{2} G_{0}^{*}+F_{0} G_{2}^{*}\right) \cos 2 \Psi[\eta(1-\kappa)(3+\cos 2 \Omega)
$$$$
\left.\times \cos 2 \omega+2(1-3 \kappa) \sin ^{2} \Omega\right]
$$$$
-\left(F_{2} G_{0}^{*}+F_{1} G_{1}^{*}+F_{0} G_{2}^{*}\right)((1-3 \kappa) \cos 2 \Omega
$$$$
\left.+2 \eta(1-\kappa) \cos 2 \omega \sin ^{2} \Omega\right)-4 \eta(1-\kappa)
$$$$
\times\left(F_{2} G_{0}^{*}+F_{0} G_{2}^{*}\right) \cos \Omega \sin 2 \Psi \sin 2 \omega,
$$

$$
\begin{aligned}
C_{3}= & 2\left(F_{2} G_{1}^{*}+F_{1} G_{2}^{*}\right) \sin \Omega \cdot[\cos \Psi \cos \Omega(1-3 \kappa-\eta \\
& \times(1-\kappa) \cos 2 \omega)+\eta(1-\kappa) \sin \Psi \sin 2 \omega],
\end{aligned}
$$

$$
C_{4}=-F_{2} G_{2}^{*}\{\cos 2 \Psi[\eta(1-\kappa)(3+\cos 2 \Omega) \cos 2 \omega+2(1
$$$$
\left.\left.-3 \kappa) \sin ^{2} \Omega\right]-4 \eta(1-\kappa) \cos \Omega \sin 2 \Psi \sin 2 \omega\right\},
$$

$$
\begin{aligned}
S_{1}= & S_{3}=2\left(F_{2} G_{1}^{*}+F_{1} G_{2}^{*}\right) \sin \Omega \cdot\{\sin \Psi \cos \Omega[1-3 \kappa \\
& -\eta(1-\kappa) \cos 2 \omega]-\eta(1-\kappa) \cos \Psi \sin 2 \omega\},
\end{aligned}
$$

$$
\begin{aligned}
S_{2}= & \left(F_{2} G_{0}^{*}+F_{0} G_{2}^{*}\right)\{\sin 2 \Psi[\eta(1-\kappa)(3+\cos 2 \Omega) \cos 2 \omega \\
& \left.+2(1-3 \kappa) \sin ^{2} \Omega\right]+4 \eta(1-\kappa) \\
& \times \cos 2 \Psi \cos \Omega \sin 2 \omega\},
\end{aligned}
$$

$$
\begin{aligned}
S_{4}= & -F_{2} G_{2}^{*}\left\{\operatorname { s i n } 2 \Psi \left[\eta(1-\kappa)(3+\cos 2 \Omega) \cos 2 \omega_{0}-2(1\right.\right. \\
& \left.\left.-3 \kappa) \sin ^{2} \Omega\right]+4 \eta(1-\kappa) \cos \Omega \cos 2 \Psi \sin 2 \omega\right\} .
\end{aligned}
$$

The above expressions were derived for detection through a polarization filter transmitting fluorescence that is polarized along the $x$-direction. In a standard set-up of defocused imaging, detection is performed without any polarizer in the detection path. The detected pattern for unpolarized detection is then given as the linear superposition

$$
\begin{aligned}
P_{\text {total }}= & P(\rho, \phi, \Psi, \Omega, \omega, \kappa, \eta, \delta f) \\
& +P(\rho, \phi-\pi / 2, \Psi-\pi / 2, \Omega, \omega, \kappa, \eta, \delta f) .
\end{aligned}
$$

Although these results look rather complex, they have several remarkable properties. Its dependence on the orientation variables $\Omega, \Psi$, and $\omega$ involves only simple trigonometric functions as shown in the expressions for the $C_{j}$ and $S_{j}$; only the integrals $F_{j}$ and $G_{j}$ depend on the coordinate $\rho$ and the defocusing $\delta f$. It is noteworthy that although $P$ includes terms varying as the third and fourth harmonic in $\phi$, the total intensity pattern $P_{\text {total }}$ includes only harmonics up to the second order. It can also be shown that a circularly degenerate dipole emitter is indistinguishable from two crossed linear dipoles of equal emission strength. Let us compare defocused imaging with standard fluorescence anisotropy measurements using a polarizing filter in the detection channel. That corresponds to integrating the fluorescence intensity over the detector's surface, i.e.,

$$
\begin{aligned}
\widetilde{P}(\Psi, \Omega, \omega, \kappa, \eta) & =\int_{0}^{\infty} d \rho \rho \int_{0}^{2 \pi} d \phi P(\rho, \phi, \Psi, \Omega, \omega, \kappa, \eta, \delta f) \\
& =\int_{0}^{\infty} d \rho \rho C_{0} .
\end{aligned}
$$

Comparing the last equation with Eq. (4) shows that fluorescence anisotropy measurements detect only information contained in $C_{0}$, but omitting all information of the higher harmonic coefficients in Eq. (4). Rotating the analyzer corresponds to changing the angle $\Psi$ in $C_{0}$. This results in an intensity variation of the form $a_{0}+a_{1} \cos 2\left(\Psi-\Psi_{0}\right)$ with some constants $a_{0}, a_{1}$, and $\Psi_{0}$. For any measured pair of values $\left\{a_{0}, a_{1}\right\}$, there exists an infinite number of corresponding parameter values $\Omega, \omega, \eta$, and $\kappa$. Thus, fluorescence anisotropy measurements cannot distinguish between, e.g., a circular dipole emitter with its dark axis inclined to the optical axis and two crossed linear dipole emitters oriented perpendicular to the optical axis but having unequal emission strengths.

Defocused images of nanocrystals were measured using a setup as described in Ref. 10, with an excitation wavelength of $514.5 \mathrm{~nm}$ and $200 \mathrm{~W} / \mathrm{cm}^{2}$ intensity. The studied sample consisted of microscope cover slides (Menzel) of $170 \mu \mathrm{m}$ thickness, on top of which $100 \mu \mathrm{l}$ of a diluted solution of the CdSe/ZnS nanocrystals (QuantumDot Corp.) in bidistilled water was spin-coated and dried, yielding a sparse distribution of nanocrystals on the surface. According to the available information on the synthesis of these nanocrystals, ${ }^{12}$ they adapt the wurtzite form of CdSe. Images of the sample were taken with exposure times of $30 \mathrm{~s}$. Image defocusing was done by moving the objective a distance $\delta f$ towards the sample.

Downloaded 21 Dec 2006 to 134.94.122.39. Redistribution subject to AIP license or copyright, see http://apl.aip.org/apl/copyright.jsp 


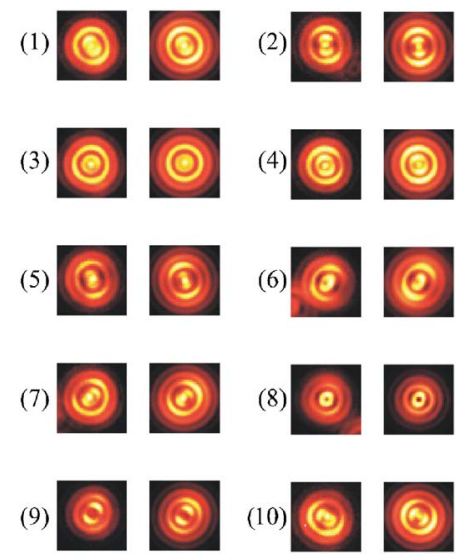

FIG. 2. (Color online) Measured and theoretically calculated defocused emission patterns for ten quantum dots. In each panel, the left image is the measured image, and the right the corresponding theoretical calculation. The model parameters used for each image were $(\delta f$ in $\mu \mathrm{m}):(1) \Omega=87.00, \omega_{0}$ $=0.00, \Psi=30.00, \kappa=0.62, \eta=0.81, \delta f=1.20 ;(2) \Omega=90.00, \omega_{0}=4.00, \Psi=$ $-173.00, \kappa=1.00 \eta=0.82, \delta f=1.20$; (3) $\Omega=2.00, \omega_{0}=0.00, \Psi=-140.00$, $\kappa=0.04 \eta=0.00, \delta f=1.21$; (4) $\Omega=1.00, \omega_{0}=48.00, \Psi=155.00, \kappa=0.24 \eta$ $=0.00, \delta f=1.17$; $(5) \Omega=87.00, \omega_{0}=173.00, \Psi=-160.00, \kappa=1.00 \eta=0.80$, $\delta f=1.25$; (6) $\Omega=84.00, \omega_{0}=69.00, \Psi=149.00, \kappa=0.44 \eta=0.70, \delta f=1.22$; (7) $\Omega=82.00, \Omega_{0}=12.00, \Psi=-47.00, \kappa=1.00 \quad \eta=0.72, \delta f=1.22$; (8) $\Omega$ $=90.00, \Omega_{0}=60.00, \Psi=-113.00, \kappa=0.16 \eta=0.00, \delta f=0.95$; (9) $\Omega=87.00$, $\omega_{0}=15.00, \Psi=-32.00, \quad \kappa=1.00 \quad \eta=0.74, \delta f=1.10 ; \quad(10) \Omega=84.00, \omega_{0}$ $=3.00, \Psi=-122.00, \kappa=1.00 \eta=0.72, \delta f=1.18$.

Figure 2 shows a comparison between measured and calculated defocused images for ten nanocrystals. When searching for the parameter values of $\Psi, \Omega, \omega, \kappa$, and $\eta$ that best reproduced the observed patterns, the defocusing value $\delta f$ was also allowed to vary slightly due to some uncertainty of its experimental value caused by mechanical drift of the microscope stage and bending of the cover slide. Parameter searching was done by a pattern matching algorithm as described in Ref. 10. Figure 2 shows that the achieved correspondence between calculated and measured images is excellent. We have evaluated images of 41 different quantum dots, and the majority of them exhibits an angular distribution of emission expected for a superposition of at least two perpendicular dipoles with different emission strength. Some of the observed patterns show a clear nonbilateral symmetry (e.g., images 6, 7, and 10 in Fig. 2) which is impossible for a purely linear dipole or a circularly degenerate dipole emitter, thus presenting clear evidence of a more complex emission behavior. The most interesting characteristic of the emission are the parameters $\eta$ and $\kappa$ describing the relative emission strength of the three possible dipoles. Figure 3 plots the observed absolute values of $|\eta|$ against the values of $\kappa$. Most quantum dots have an emission characteristic close to that of two crossed linear dipoles with an emission strength ratio of ca. 0.7. When inspecting Fig. 3, it should be noted that the parameter pair $\{|\eta|, \kappa\}$ is not an unambiguous characterization: for example, the value pair $\{1, \xi\}$ describes a physically identical situation as $\{|1-2 \xi|, 0\}$, for any value $0 \leqslant \xi \leqslant 1$. The reason for the observed emission behavior is probably due to the shapes of the nanocrystals. With increasing elongation of the shape along the extraordinary axis of the wurtzite-type crystal structure, the dipole transition along that axis becomes dominant, but a perpendicular dipole component still remains. Another explanation could be breaking of the hexagonal symmetry of the nanocrystals, destroying the degen-

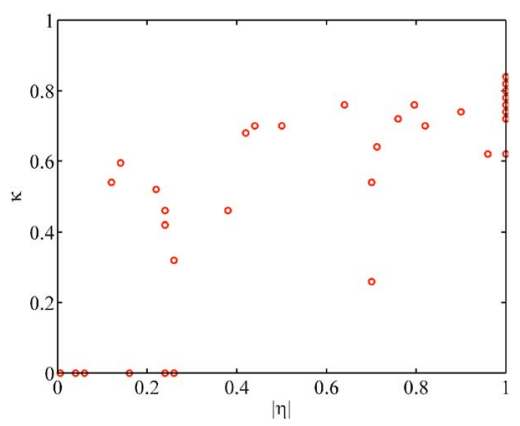

FIG. 3. (Color online) Relation between the determined values of $|\eta|$ and $k$ for the 41 evaluated quantum dots. Value pairs with $\kappa=0$ and $|\eta|$ close to zero correspond to a circular degenerate dipole emitter, values with $\kappa=1$ to a purely linear dipole emitter.

eracy of states perpendicular to the extraordinary axis. The possibility of having more than a single fluorescing quantum dot within one particle could be excluded on the basis of fluorescence antibunching measurements. ${ }^{13}$

In summary, we have applied defocused imaging for observing the angular distribution of fluorescent quantum dots. It was observed that individual quantum dots show differing angular distributions of emission, the majority exhibiting an emission of elliptically degenerate dipole emitters.

We thank Thomas Ruckstuhl for many inspiring discussions. We are grateful to Benjamin Kaupp for his general support of our work.

${ }^{1}$ M. Nirmal, B. O. Dabbousi, M. G. Bawendi, J. J. Macklin, J. K. Trautman, T. D. Harris, and L. E. Brus, Nature (London) 383, 802 (1996); S. A. Empedocles, D. J. Norris, and M. G. Bawendi, Phys. Rev. Lett. 77, 3873 (1996); G. Schlegel, J. Bohnenberger, I. Potapova, and A. Mews, ibid. 88, 137401 (2002); X. Brokmann, L. Coolen, M. Dahan, and J. P. Hermier, ibid. 93, 107403 (2004).

${ }^{2}$ A. L. Efros, Phys. Rev. B 46, 7448 (1992); S. A. Empedocles, R. Neuhauser, and M. G. Bawendi, Nature (London) 399, 126 (1999).

${ }^{3}$ S. A. Empedocles and M. G. Bawendi, Science 278, 2114 (1997); J. Tittel, W. Göhde, F. Koberling, A. Mews, A. Kornowski, H. Weller, A. Eychmüller, and T. Basché, Ber. Bunsenges. Phys. Chem. 101, 1626 (1997).

${ }^{4}$ A. L. Efros, M. Rosen, M. Kuno, M. Nirmal, D. J. Norris, and M. Bawendi, Phys. Rev. B 54, 4843 (1996); J. J. Shiang, A. V. Kadavanich, R. K. Grubbs, and A. P. Alivisatos, J. Phys. Chem. 99, 17417 (1995); J. Hu, L. S. Li, W. Yang, L. Manna, L. W. Wang, and A. P. Alivisatos, Science 292, 2060 (2001).

${ }^{5}$ M. Böhmer and J. Enderlein, J. Opt. Soc. Am. B 20, 554 (2003).

${ }^{6}$ A. P. Bartko and R. M. Dickson, J. Phys. Chem. B 103, 3053 (1999); 103, 11237 (1999).

${ }^{7}$ A. P. Bartko, L. A. Peyser, R. M. Dickson, A. Mehta, T. Thundat, R. Bhargava, and M. D. Barnes, Chem. Phys. Lett. 358, 459 (2002).

${ }^{8}$ A. P. Bartko, K. Xu, and R. M. Dickson, Phys. Rev. Lett. 89, 026101 (2002).

${ }^{9}$ W. Schroeyers, R. Vallee, D. Patra, J. Hofkens, S. Habuchi, T. Vosch, M. Cotlet, K. Müllen, J. Enderlein, and F. C. De Schryver, J. Am. Chem. Soc. 126, 14310 (2004).

${ }^{10}$ D. Patra, I. Gregor, and J. Enderlein, J. Phys. Chem. A 108, 6836 (2004). ${ }^{11}$ W. Lukosz, J. Opt. Soc. Am. 69, 1495 (1979); J. Enderlein, T. Ruckstuhl, and S. Seeger, Appl. Opt. 38, 724 (1999).

${ }^{12}$ B. O. Dabbousi, J. Rodriguez-Viejo, F. V. Mikulec, J. R. Heine, H. Mattoussi, R. Ober, K. F. Jensen, and M. G. Bawendi, J. Phys. Chem. B 101, 9463 (1997); X. Wu, H. Liu, J. Liu, K. N. Haley, J. A. Treadway, J. P. Larson, N. Ge, F. Peale, and M. P. Bruchez, Nat. Biotechnol. 2, 41 (2003). ${ }^{13}$ W. P. Ambrose, P. M. Goodwin, J. Enderlein, D. J. Semin, J. C. Martin, and R. A. Keller, Chem. Phys. Lett. 269, 365 (1997); B. Lounis, H. A. Bechtel, D. Gerion, P. Alivisatos, and W. E. Moerner, ibid. 329, 399 (2000). 